\title{
Newly diagnosed incident dizziness of older patients: a follow-up study in primary care
}

\author{
Julia Sczepanek', Birgitt Wiese ${ }^{2}$, Eva Hummers-Pradier ${ }^{1}$ and Carsten Kruschinski ${ }^{1 *}$
}

\begin{abstract}
Background: Dizziness is a common complaint of older patients in primary care, yet not much is known about the course of incident dizziness. The aim of the study was to follow-up symptoms, subjective impairments and needs of older patients ( $\geq 65$ ) with incident dizziness and to determine predictors of chronic dizziness. Furthermore, we analysed general practitioners' (GPs') initial diagnoses, referrals and revised diagnoses after six months.

Methods: An observational study was performed in 21 primary care practices in Germany, including a four-week and six-month follow-up. A questionnaire comprising characteristic matters of dizziness and a series of validated instruments was completed by 66 participants during enrolment and follow-up (after 1 month and 6 months). After six months, chart reviews and face-to-face interviews were also performed with the GPs.
\end{abstract}

Results: Mean scores of dizziness handicap, depression and quality of life were not or only slightly affected, and did not deteriorate during follow-up; however, 24 patients (34.8\%) showed a moderate or severe dizziness handicap, and $43(62.3 \%)$ showed a certain disability in terms of quality of life at the time of enrolment. In multivariate analysis, $\mathrm{n}=44$ patients suffering from chronic dizziness (dependent variable, i.e. relapsing or persistent at six months) initially had a greater dizziness handicap (OR $1.42,95 \% \mathrm{Cl} 1.05-1.47$ ) than patients with transient dizziness. GPs referred $47.8 \%$ of the patients to specialists who detected two additional cases of benign paroxysmal positional vertigo (BPPV).

Conclusions: New-onset dizziness relapsed or persisted in a considerable number of patients within six months. This was difficult to predict due to the patients' heterogeneous complaints and characteristics. Symptom persistence does not seem to be associated with deterioration of the psychological status in older primary care patients. Management strategies should routinely consider BPPV as differential diagnosis.

\section{Background}

Dizziness is a frequent complaint of older patients in primary care. Its point prevalence increases with age up to a total of $30 \%$ of people 65 years of age and older [1-4]. For the general practitioner (GP) two aims are crucial: to exclude a life-threatening or treatable specific disease and to identify a chronic development of dizziness. As to exclusion of life-threatening diseases, Bird et al. [5] found that GPs did not fail to refer the rare urgent cases. Altogether, more than one in six patients were referred inappropriately, which was more common in older people. In other studies, serious causes of dizziness were not overlooked [6-8]. However, these studies did not concentrate on the specific situation of older patients.

\footnotetext{
* Correspondence: kruschinski.carsten@mh-hannover.de

${ }^{1}$ Institute of General Practice, Hannover Medical School, Hannover, Germany Full list of author information is available at the end of the article
}

The diversity of possible aetiologies concerning multiple organ systems and associated risk factors yielded the hypothesis that dizziness represents a chronic multifactorial geriatric syndrome $[4,9,10]$. For many older patients especially the deterioration of spatial perception and its concomitant features produce a debilitating experience impairing health-related quality of life [11]. Accordingly, studies revealed associations between anxiety, depressive and somatoform disorders as well as restrictions in daily life and social activities in chronic dizziness [3,11-13].

A considerable number of early studies conclude with the requirement for prospective data, as little is known about the dizziness-associated alterations of patient characteristics (e.g. dizziness handicap, patients' needs) in relation to the time-dependent course of the symptom [14-16]. Considering this together with the two abovementioned aspects that the GP is mostly concerned

\section{Biomed Central}


about, we initially aimed to investigate the development of incident dizziness, the associated patients' impairments and needs, and to assess predictors of chronic dizziness. In parallel, we wanted to analyse GPs' preliminary diagnoses, referrals and possible work-up diagnoses.

\section{Methods}

\section{Design of the study and enrolment}

The study design was a prospective observational followup study. Participants were consecutively registered in 21 primary care practices in the city and region of Hannover, Germany. Inclusion criteria were an age of at least 65 years and incident dizziness (which had been present for less than six months and not yet shown to another doctor) as main reason for the encounter. Exclusion criteria comprised insufficient command of the German language, dementia, or terminal diseases.

All participants gave informed consent. The study was approved by the Ethical Committee of Hannover Medical School (number 4291).

\section{Questionnaire design: Assessment of patients' impairments and needs}

At the time of enrolment, patients filled in a standardised questionnaire containing sociodemographic parameters and a detailed description of the dizziness symptoms. This included the duration, the classification according to Drachman and Hart [17] (presyncope, vertigo, disequilibrium or lightheadedness/other), triggers and concomitant symptoms. The Dizziness Handicap Inventory (DHI, [18]) was used to assess the degree of disability associated with any cause of dizziness. The Dizziness Needs Assessement (DiNA, [19]) was used to assess wishes and needs of the patients concerning their dizziness complaints. Anxiety was assessed using the following question (complying with an established screening instrument for anxiety [20]; dichotomous response: yes/no): "In the past four weeks, have you been compromised by anxiety or a feeling of being emotionally out of balance?" The Geriatric Depression Scale (GDS, [21]) was applied as a basic screening for depression in older people, and the 12-item Short Form Health Survey (SF-12, [22]) was used to analyse quality of life. Activities of Daily Living (ADL, [23]) were assessed, including basic abilities such as eating and drinking, and more complex instrumental abilities (iADL) such as doing the housework. Some of the instruments were slightly modified or culturally adapted according to the specific circumstances in Germany. The explanation of the resulting scores is displayed in the table legends.

Participants filled in a questionnaire containing the same instruments one month and six months after the first consultation. Moreover, the follow-up questionnaires contained information about the persistence and intensity of the symptom. Patients were phoned or visited in order to complete missing data.

\section{Chart Review: Assessment of the doctors' diagnoses}

After six months, each of the surgeries were visited in order to perform a chart review of all patients by interviewing each GP. The ICD (International Classification of Diseases)-10-code or "free" documentation of how the GP had initially diagnosed the dizziness was specified in a face-to-face interview. Here, GPs expressed their assumptions of the aetiology of dizziness. Information on possible causes of dizziness was actively requested according to a predefined check-list. Moreover, referrals to specialists as well as the corresponding follow-up diagnoses were documented.

\section{Statistical Analysis}

All statistical analyses were performed using the SPSS statistics software (version 17.0). For analysis of the development of the patients' impairments and needs over time, the non-parametric Friedman test was used to explore differences of test scores and Likert scale items (DiNA, [19]).

For further analysis, all patients were divided into two groups: temporary dizziness (present only at the time of inclusion or persisting at the four-week follow-up but not at six months), and chronic dizziness, i.e. relapsing (not present at the four-week follow-up, but recurring at the six-month follow-up) or persistent (dizziness present at all three time points of investigation). To explore differences of test score results and Likert-scale items between the temporary and chronic dizziness groups at each time point, the Mann-Whitney-U-Test was used.

Multivariate logistic regression analysis was used to identify predictors of chronic dizziness. For inclusion in the model, variables were selected on the basis of significance taking into account the clinical relevance of the differences and the literature. For example, "depression" is a relevant co-morbidity in chronic dizziness [13] and was therefore included. Univariate and multivariate Odds Ratios (OR) with their 95\% confidence intervals (CI) were calculated for all variables.

For all statistical analyses, variables were considered significant if $\mathrm{p}<0.05$.

\section{Results}

\section{Sociodemographic characteristics}

We prospectively identified 77 patients presenting at their GPs' with a chief complaint of incident dizziness. Eight patients did not fulfil the inclusion criteria due to age ( 1 patient) or the duration of dizziness ( 7 patients). Three patients were not followed up and thus completed questionnaires from all three time points were obtained for 66 patients. The mean age of all 69 participating 
patients with dizziness was 76.19 years $(\mathrm{SD}=6.64$ years, range 65-95). The majority of patients were female $(\mathrm{n}=$ $48,69.6 \%)$. The characteristics of dizziness are shown in table 1.

\section{Psychological/functional impact and patients' needs in terms of dizziness during follow-up}

The course of dizziness and its associated implications were investigated over the 6-month period (table 2). Most mean instrument scores did not indicate pathological

Table 1 Characteristics of dizziness

\begin{tabular}{|c|c|c|}
\hline & $\mathrm{n}$ & $\%$ \\
\hline \multicolumn{3}{|l|}{ Type of dizziness* } \\
\hline unsteadiness & 45 & 65.2 \\
\hline vertigo & 32 & 46.4 \\
\hline fainting & 11 & 15.9 \\
\hline other e.g. & 12 & 19.4 \\
\hline feeling of swaying from one side to the other & 38 & 55.9 \\
\hline lift feeling & 11 & 15.9 \\
\hline \multicolumn{3}{|l|}{ Duration } \\
\hline seconds & 25 & 41.0 \\
\hline minutes to hours & 19 & 31.1 \\
\hline several hours & 10 & 16.4 \\
\hline continuous & 7 & 11.5 \\
\hline \multicolumn{3}{|l|}{ Dizziness eliciting situations*† } \\
\hline getting up from lying or sitting & 39 & 56.5 \\
\hline bending down & 36 & 52.2 \\
\hline head turning & 23 & 33.3 \\
\hline rotatory movement & 21 & 30.4 \\
\hline lying down & 19 & 27.5 \\
\hline walking & 17 & 24.6 \\
\hline standing & 13 & 18.8 \\
\hline walking on uneven ground & 13 & 18.8 \\
\hline darkness & 11 & 15.9 \\
\hline lying on one side & 9 & 13.0 \\
\hline Concomitant symptoms*† & 46 & 66.7 \\
\hline nausea & 24 & 34.8 \\
\hline tinnitus & 18 & 26.1 \\
\hline headache & 16 & 23.2 \\
\hline tachycardia & 12 & 17.4 \\
\hline weakness (extremities) & 9 & 13.0 \\
\hline muscle tremor & 8 & 11.6 \\
\hline vomiting & 8 & 11.6 \\
\hline dyspnoea & 7 & 10.1 \\
\hline numbness (extremities) & 7 & 10.1 \\
\hline perspiration & 7 & 10.1 \\
\hline visual disturbance & 7 & 10.1 \\
\hline
\end{tabular}

${ }^{*}$ more than one answer possible.

† symptoms listed only if present in more than $10 \%$ of cases. values at enrolment (T0), except a moderate restriction in terms of quality of life (SF-12). However at T0, the proportion of "disabled" patients (SF-12, score less than 51) was $43 / 69$ (62.3\%), and the proportion of patients showing a moderate or severe dizziness handicap (modified DHI, score 26 or more) was $24 / 69$ (34.8\%).

The mean scores of most instruments were relatively stable over time. However, in terms of the dizziness handicap there was a difference depending on the instrument used. The DiNA (patients' needs), in which the "handicap during acute episodes of dizziness" can be differentiated from a "general dizziness handicap", revealed that the handicap during acute dizziness was high at the beginning and decreased significantly over time. This was different from the "general dizziness handicap" (DiNA) as well as from the handicap as measured by the DHI.

Patients considered it very important to know the cause of the dizziness (DiNA), which remained stable over time, and to be taken seriously by their doctor ("empathy of doctor"), though this aspect decreasing significantly over time. Anxiety screening was positive in $41.2 \%$ of patients at the time point of first assessment.

\section{Comparing patients suffering from temporary or chronic dizziness}

Apart from the intraindividual follow-up of the patients' impairments and needs (table 2), patients were analysed separately according to whether they belonged to the temporary $(n=22)$ or chronic dizziness group (i.e. the dizziness persisted after the 6 months follow-up, $n=$ 44 ), at each time point of assessment (table 3). Although neither group achieved true "pathological" mean values, patients suffering from chronic dizziness manifested stronger impairments in terms of dizziness handicap (DHI) and ADL than those of the temporary group at the time of enrolment as well as during follow-up (ADL). Considering the patients' needs (DiNA), the perceived GP's capability of helping with their dizziness ("help of doctor") was rated significantly higher in the temporary group in comparison to the chronic group at the time of first assessment.

Moreover, patients' responses were evaluated using logistic regression analysis in order to define predictors of chronic dizziness. For this purpose, "chronic dizziness" (yes/no) was defined as the dependent variable (outcome). For the covariates included (see table 4) only the scores of the time point of inclusion (T0) were considered, as we were interested in whether these variables could help the GP at the first time of encounter to anticipate whether dizziness would become chronic. In this multivariate analysis, a greater handicap, as measured by means of the DHI, was associated with chronic dizziness. A patient's perception that the GP would be 
Table 2 Development of the different instrument scores during follow-up (mean \pm SD)

\begin{tabular}{|c|c|c|c|c|c|}
\hline Instrument & Explanation/Item & TO & T1 & $\mathrm{T} 2$ & $\mathrm{P}^{*}$ \\
\hline DHI total† & Dizziness handicap & $26.68 \pm 13.27$ & $22.95 \pm 15.12$ & $24.32 \pm 15.24$ & 0.055 \\
\hline physical & (subscale) & $7.05 \pm 3.59$ & $6.00 \pm 3.97$ & $6.21 \pm 3.91$ & 0.195 \\
\hline functional & (subscale) & $11.42 \pm 7.41$ & $10.68 \pm 8.44$ & $11.57 \pm 8.55$ & 0.164 \\
\hline emotional & (subscale) & $8.21 \pm 5.44$ & $6.26 \pm 6.10$ & $6.53 \pm 5.97$ & 0.036 \\
\hline SF12 & Quality of life & $47.03 \pm 11.61$ & $49.41 \pm 10.08$ & $48.64 \pm 9.94$ & 0.639 \\
\hline GDS & Depression & $3.19 \pm 2.90$ & $3.08 \pm 2.94$ & $2.63 \pm 2.76$ & 0.174 \\
\hline ADL total & Activities of daily living & $39.63 \pm 4.05$ & $39.72 \pm 4.08$ & $39.53 \pm 3.29$ & 0.407 \\
\hline basic & (subscale) & $15.23 \pm 1.47$ & $15.11 \pm 1.62$ & $15.28 \pm 1.13$ & 0.620 \\
\hline instrumental & (subscale, e.g. financial tasks) & $20.67 \pm 2.52$ & $20.84 \pm 2.30$ & $20.56 \pm 2.15$ & 0.491 \\
\hline \multirow[t]{7}{*}{ DiNA } & Importance of knowing the cause & $4.91 \pm 1.42$ & $4.82 \pm 1.38$ & $4.91 \pm 1.18$ & 0.465 \\
\hline & Risk of falling & $3.47 \pm 1.52$ & $3.09 \pm 1.42$ & $3.15 \pm 1.42$ & 0.300 \\
\hline & Effectiveness of self-help measures & $3.80 \pm 1.47$ & $4.30 \pm 1.42$ & $4.15 \pm 1.35$ & 0.368 \\
\hline & Handicap during acute dizziness & $4.03 \pm 1.52$ & $3.76 \pm 1.55$ & $3.49 \pm 1.57$ & 0.058 \\
\hline & General dizziness handicap & $3.03 \pm 1.42$ & $2.72 \pm 1.34$ & $2.67 \pm 1.35$ & 0.028 \\
\hline & Empathy of doctor & $5.22 \pm 1.20$ & $4.69 \pm 1.37$ & $4.25 \pm 1.52$ & $<0.001$ \\
\hline & Help of doctor & $2.83 \pm 1.39$ & $3.07 \pm 1.60$ & $2.87 \pm 1.50$ & 0.630 \\
\hline
\end{tabular}

T0, T1, T2 represent the three time points of assessment (see legend).

TO first time point of assessment.

T1 second time point of assessment (four-week follow-up).

$\mathrm{T} 2$ third time point of assessment (six-month follow-up).

* Differences of mean test score and Likert scale items (DiNA) comparing the three time points were tested using the Friedman test.

† scores: DHI/modified (maximum score [= highest handicap]: $88, \geq 27$ : moderate or severe handicap; adapted according to [31]), SF-12 ( $\geq 51$ : "no disability", $\leq 30$ : "severe disability"), GDS (<6: "no depression", > 6 and < 11: "mild to moderate", $\geq 11$ : "severe"), ADL/modified (maximum score [= lowest handicap]: 42 [instrumental (iADL): 22, ADL: 16, continence: 4]), DiNA (Likert scales 1-6 [growing with increasing numbers]).

unable to help with respect to their dizziness ("How much can your doctor help you in terms of your dizziness?") at T0, was also associated with chronic dizziness, and a positive anxiety screening was negatively associated with chronic dizziness.

\section{GPs' diagnoses and referrals}

In parallel to the description of the patients' perspective, we also identified the GPs' view by describing their preliminary and work-up diagnoses after referral (table 5). In most cases, $(\mathrm{n}=20,29.0 \%)$, a multicausal aetiology (two or more possible causes) of dizziness had initially been assumed by the GPs. During the six-month followup period, $\mathrm{n}=33(47.8 \%)$ of the participants were referred to at least one specialist, including three patients that were sent to hospital because of symptom severity (suspicion of TIA, hypertensive crisis) or psychosocial reasons. In 6/33 cases (18.2\%) the specialists' diagnoses were explicitly different from those of the GP, including two patients with newly diagnosed BPPV.

\section{Discussion}

\section{Summary of main findings}

The present study demonstrates that a considerable aspect of older patients suffering from newly diagnosed, incident dizziness was associated with the dizziness handicap and reduced quality of life. However, mean scores of most outcomes (including dizziness handicap, quality of life, depression, activities of daily living) were only slightly affected and relatively stable during the six months of follow-up. This as well as the heterogeneous nature of the dizziness complaints in our sample made it difficult to clearly identify variables able to predict chronic dizziness. As to the patients' needs, knowing the cause of dizziness was repeatedly rated as very important. The empathy of the doctor was perceived as high, but this decreased over the study period. As to the GPs' management strategies, almost half of the patient study population was referred to a specialist. In two cases, benign paroxysmal positional vertigo (BPPV) was diagnosed only after specialist consultation.

\section{Strengths and limitations of the study}

Whereas previous studies have mainly considered the overall outcome of dizziness $[6,8,24]$, we prospectively assessed over time the intraindividual impairment of patients of a specific age group using the same set of validated instruments. However, it is necessary to mention that the sample size of our cohort was relatively small. This can be explained by closely framed inclusion criteria, which made recruitment of patients challenging. Obviously, incident dizziness was much less common in older patients than had previously been expected; however, the study had not been designed to allow 
Table 3 Instrument scores (mean \pm SD) comparing temporarily dizzy (not any more present after six months, $\mathbf{n}=\mathbf{2 2}$ ) and chronically dizzy (still present after six months, $n=44$ ) patients at the three time points of investigation

\begin{tabular}{|c|c|c|c|c|c|c|c|c|c|c|}
\hline Instrument & Explanation/Item & $\begin{array}{c}\text { T0 } \\
\text { temporary }\end{array}$ & $\begin{array}{c}\text { T0 } \\
\text { chronic }\end{array}$ & $\mathrm{P}^{*}$ & T1 temporary & $\begin{array}{c}\text { T1 } \\
\text { chronic }\end{array}$ & $\mathrm{P}^{*}$ & T2 temporary & $\begin{array}{c}\text { T2 } \\
\text { chronic }\end{array}$ & $\mathrm{P}^{*}$ \\
\hline$\overline{\mathrm{DHI}}$ & Dizziness handicap & $15.27 \pm 14.42$ & $25.36 \pm 13.90$ & 0.004 & $18.80 \pm 17.64$ & $22.95 \pm 15.12$ & 0.258 & n.a. & $22.59 \pm 15.14$ & n.a. \\
\hline SF12 & Quality of life & $49.09 \pm 11.63$ & $45.82 \pm 11.34$ & 0.294 & $51.76 \pm 9.75$ & $48.5 \pm 10.16$ & 0.279 & $51.4 \pm 7.63$ & $47.39 \pm 10.67$ & 0.178 \\
\hline GDS & Depression & $2.41 \pm 2.15$ & $3.66 \pm 3.14$ & 0.138 & $2.52 \pm 2.82$ & $3.32 \pm 2.96$ & 0.265 & $2.10 \pm 2.27$ & $2.86 \pm 2.95$ & 0.287 \\
\hline ADL & Activities of daily living & $41.14 \pm 2.17$ & $38.95 \pm 4.50$ & 0.001 & $40.86 \pm 2.33$ & $39.18 \pm 4.58$ & 0.013 & $40.67 \pm 2.22$ & $39.05 \pm 3.58$ & 0.045 \\
\hline \multirow[t]{7}{*}{ DiNA } & Importance of knowing the cause & $5.18 \pm 1.44$ & $5.12 \pm 1.31$ & 0.769 & $5.20 \pm 1.32$ & $4.67 \pm 1.41$ & 0.309 & n.a. & $5.02 \pm 1.18$ & n.a. \\
\hline & Risk of falling & $3.00 \pm 1.61$ & $3.37 \pm 1.57$ & 0.317 & $2.80 \pm 1.87$ & $3.08 \pm 1.38$ & 0.356 & n.a. & $3.07 \pm 1.57$ & n.a. \\
\hline & Effectiveness of self-help measures & $4.07 \pm 1.39$ & $3.52 \pm 1.60$ & 0.268 & $4.25 \pm 1.83$ & $4.11 \pm 1.45$ & 0.659 & n.a. & $3.75 \pm 1.72$ & n.a. \\
\hline & Handicap during acute dizziness & $4.29 \pm 1.55$ & $3.98 \pm 1.58$ & 0.392 & $3.70 \pm 1.34$ & $3.76 \pm 1.55$ & 0.905 & n.a. & $3.39 \pm 1.56$ & n.a. \\
\hline & General dizziness handicap & $2.76 \pm 1.55$ & $3.09 \pm 1.49$ & 0.360 & $2.40 \pm 0.97$ & $2.72 \pm 1.34$ & 0.471 & n.a. & $2.52 \pm 1.34$ & n.a. \\
\hline & Empathy of doctor & $5.62 \pm 0.59$ & $5.32 \pm 1.14$ & 0.552 & $5.30 \pm 1.06$ & $4.69 \pm 1.37$ & 0.180 & n.a. & $4.27 \pm 1.58$ & n.a. \\
\hline & Help of doctor & $4.26 \pm 1.56$ & $2.93 \pm 1.46$ & 0.004 & $3.33 \pm 1.80$ & $3.13 \pm 1.61$ & 0.729 & n.a. & $3.07 \pm 1.59$ & n.a. \\
\hline
\end{tabular}

Scores are explained in the legend of table 2.

To first time point of assessment

T1 second time point of assessment (four-week follow-up)

T2 third time point of assessment (six-month follow-up)

* Differences of mean scores and Likert-scale items between the temporary and chronic group at each time point were tested using the Mann-Whitney-U-Test

n.a. not applicable 
Table 4 Predictors for chronic dizziness including univariate and adjusted $\mathrm{OR}$ and $95 \% \mathrm{Cl}$.

\begin{tabular}{lcccccc}
\hline & \multicolumn{3}{c}{ univariate analysis } & \multicolumn{3}{c}{ multivariate analysis } \\
\hline & OR & $\mathbf{9 5 \%} \mathbf{C l}$ & $\mathbf{P}$ & $\mathbf{O R}$ & $\mathbf{9 5 \%} \mathrm{Cl}$ & $\mathbf{P}$ \\
\hline age & 1.07 & $0.97-1.16$ & 0.196 & 1.09 & $0.91-1.32$ & 0.352 \\
female gender & 0.87 & $0.25-3.03$ & 0.824 & 0.51 & $0.04-6.02$ & 0.591 \\
Type of dizziness & & & & & & \\
vertigo & 0.48 & $0.15-1.53$ & 0.214 & 1.27 & $0.12-14.05$ & 0.846 \\
fainting & 5.93 & $0.69-$ & 0.104 & 13.78 & $0.41-$ & 0.144 \\
& & 50.70 & & & 465.80 & \\
unsteadiness & 1.47 & $0.43-5.05$ & 0.538 & 0.24 & $0.01-4.92$ & 0.355 \\
other form & 0.76 & $0.19-3.05$ & 0.697 & 0.12 & $0.01-2.51$ & 0.170 \\
Instruments & & & & & & \\
GDS*-Score & 1.27 & $0.99-1.62$ & 0.061 & 1.55 & $0.80-3.00$ & 0.190 \\
anxiety (screening) & 2.98 & $0.91-9.74$ & 0.071 & 0.01 & $0.00-0.43$ & 0.016 \\
DHIt-Score & 1.12 & $1.04-1.20$ & 0.002 & 1.24 & $1.05-1.47$ & 0.013 \\
help of doctor & 0.54 & $0.35-0.84$ & 0.003 & 0.37 & $0.15-0.94$ & 0.037 \\
(DiNAf) & & & & & & \\
\hline
\end{tabular}

* Geriatric Depression Scale.

† Dizziness Handicap Inventory.

‡ Dizziness Needs Assessment. identification of exact incidence rates. According to the participating GPs' experience, older patients did not usually indicate a starting point of their illness, but mentioned their symptoms only occasionally, if at all. For these reasons, we cannot be sure whether cases have really been included consecutively. In terms of diagnoses we relied -except in the referred cases- on the face validity of the GPs' diagnostic label. This was done in order to reduce the influence of the effects of a study protocol (Hawthorne effect) in favour of a description of the GPs' "usual" behaviour.

\section{Comparison with existing literature}

In our study, chronically dizzy patients were more handicapped than temporarily dizzy patients; this was reflected by the fact that higher initial dizziness handicap scores (found from the questionnaires completed at the time of enrolment) tended to predict persistence of the symptom in our multivariate analyses. The majority of patients had a somewhat diminished quality of life in our study sample, however without a significant difference between

Table 5 GPs' preliminary diagnoses and referrals including specialists' (work-up) diagnoses

\begin{tabular}{|c|c|c|c|c|c|c|}
\hline $\begin{array}{l}\text { Provisional } \\
\text { diagnosis by } \\
\text { GP }\end{array}$ & $\begin{array}{l}\text { No. of } \\
\text { patients } \\
\text { (\%) }\end{array}$ & & & & & \\
\hline & & $\begin{array}{l}\text { Patients } \\
\text { referred* }\end{array}$ & & & & \\
\hline & & & $\begin{array}{l}\text { Did not visit } \\
\text { specialist }\end{array}$ & $\begin{array}{l}\text { Confirmation of } \\
\text { GPs' diagnoses }\end{array}$ & $\begin{array}{l}\text { No attributable } \\
\text { causes found }\end{array}$ & $\begin{array}{l}\text { GPs' diagnoses if different from specialists' } \\
\text { diagnoses }\end{array}$ \\
\hline multicausalt & $20(29.0)$ & 10 & 0 & $\begin{array}{l}1 \text { BPPV } \\
1 \text { central }\end{array}$ & 5 & $\begin{array}{l}1 \text { BPPV } \\
1 \text { orthostatic dysregulation } \\
1 \text { exclusion of presumed peripheral vestibular } \\
\text { vertigo }\end{array}$ \\
\hline cardiogenic & $9(13.0)$ & 2 & 0 & 0 & 1 & 1 cervicogenic \\
\hline cervicogenic & $9(13.0)$ & 4 & 1 & 3 & 0 & 0 \\
\hline symptomatic $\neq$ & $4(5.8)$ & 2 & 1 & 0 & 0 & 1 peripheral vestibular \\
\hline $\begin{array}{l}\text { peripheral } \\
\text { vestibular }\end{array}$ & $6(8.7)$ & 4 & 0 & 3 & 1 & $\begin{array}{l}\text { (in two cases specialists mentioned a central } \\
\text { cause as additional differential diagnosis) }\end{array}$ \\
\hline BPPV & $6(8.7)$ & 2 & 2 & 0 & 0 & 0 \\
\hline $\begin{array}{l}\text { vestibular } \\
\text { neuritis }\end{array}$ & $4(5.8)$ & 3 & 0 & 0 & 2 & $1 \mathrm{BPPV}$ \\
\hline psychogenic & $2(2.9)$ & 0 & - & - & - & - \\
\hline $\begin{array}{l}\text { Ménière's } \\
\text { disease }\end{array}$ & $1(1.4)$ & 1 & 0 & 1 & 0 & 0 \\
\hline central & $1(1.4)$ & 1 & 0 & 0 & 1 & 0 \\
\hline none & $7(10.1)$ & 4 & 3 & 0 & 1 & 0 \\
\hline Total n (\%) & $69(100.0)$ & $\begin{array}{l}33 / 69 \\
(47.8)\end{array}$ & $7 / 33(21.2)$ & 9/33 (27.3) & 11/33 (33.3) & 6/33 (18.2) \\
\hline
\end{tabular}

* Patients were referred to the following specialists (more than one referral per patient possible): otorhinolaryngologist $(n=20)$, neurologist $(n=10)$, orthopaedic surgeon $(n=7)$, cardiologist $(n=7)$, hospital $(n=3)$.

$\dagger$ at least two possible differential diagnoses.

$\neq$ e.g. during infection. 
temporarily and chronically affected patients. Other studies have shown similar or even more pronounced results in terms of handicap and a generally lower quality of life in dizzy patients $[3,11,25]$, the latter being worse dependent on symptom duration. Similarly, Hsu et al. [11] and Tinetti et al. [13] found a correlation between symptom frequency and indicators of impaired functional and psychological outcome.

Former studies also revealed a high prevalence of psychological diagnoses [6,7] and a strong association between dizziness and psychological disorders such as depression and anxiety $[3,7,12,13]$. Similarly, in our study, self-reported anxiety was frequent initially. However, our patients (unlike those in other studies), did not suffer from depression at any time.

In general, comparability with other investigations was difficult: Most studies were cross-sectional rather than longitudinal, or patients were recruited in different settings (i.e. population-based). Another reason could be that a variety of assessment scales were used and, therefore, discrepancies were to be expected. As assessed by our own qualitative approach [26], the concept of dizziness handicap should contain both acute and a more general impairment. By considering both in the present study using the DiNA, handicap during acute episodes could be verified, which is an aspect that goes beyond the scope of the well-established DHI. The fact that the "importance of knowing the cause" was rated high and the perceived "empathy" decreased over time confirms findings of one of our previous studies [19] and might reflect a possible lack of patient-centeredness.

In terms of diagnostic strategies, a number of studies emphasised GPs' diagnoses based on medical history, clinical examination and follow-up as reliable and efficient $[8,15,24,27]$. However, Bird et al. [5] suggested further training of GPs was required because of inadequate referral for specialist consultation. Certainly, it is worth noting that in comparison to our data, Bird's study population was on average eight years younger, and the referral rate amounted to only $16 \%$. The fact that $29 \%$ of our dizzy patients were not assigned a specific diagnosis is in accordance with other descriptions of a relatively high frequency of "unspecific dizziness" in primary care [28] or with understanding dizziness as a geriatric syndrome [4]. Our study confirmed BPPV as a relatively common [8], but frequently underestimated cause of dizziness among older people in primary care [29], although it can be easily assessed and effectively treated [30].

\section{Conclusions}

Plausible factors indicating persistence of new-onset dizziness could not be identified, except an initially higher overall dizziness handicap. A patient-centred approach integrating functional impairments as well as patients' priorities, e.g. their need to understand causes, seems valuable in a situation where causative treatment is often not possible. This approach would also integrate psychosocial factors such as anxiety. Although dizziness is often understood as a multicausal geriatric syndrome in primary care, diagnostic algorithms would be useful and need to be evaluated so as not to miss treatable causes such as BPPV.

\section{Abbreviations}

GP: General Practitioner; OR: Odds Ratio; Cl: Confidence Interval; BPPV: Benign Paroxysmal Positional Vertigo; DHI: Dizziness Handicap Inventory; DiNA: Dizziness Needs Assessment; GDS: Geriatric Depression Scale; SF-12: 12-item Short Form Health Survey; ADL: Activities of Daily Living; iADL: Instrumental Activities of Daily Living; ICD: International Classification of Diseases; SPSS: Statistical Package for the Social Sciences; SD: Standard Deviation; TIA: Transient Ischaemic Attack;

\section{Acknowledgements}

The authors are grateful to all participating patients and their general practitioners. Moreover, we acknowledge our documentation assistant Annette Broll for data administration and Sheila Fryk as well as Susan Smith for correcting the English. This study forms part of a research project funded by the German Ministry of Education and Research (grant number

01GK0611). The article processing charge has been funded by a programme of the Deutsche Forschungsgemeinschaft (DFG): "Open access publication".

\section{Author details}

${ }^{1}$ Institute of General Practice, Hannover Medical School, Hannover, Germany. ${ }^{2}$ Centre for Biometry, Medical Informatics and Medical Technology, Hannover Medical School, Hannover, Germany.

\section{Authors' contributions}

JS carried out the chart review, undertook the statistical analysis and interpretation of the data and drafted the manuscript. CK was responsible for the study concept and design, data analysis and interpretation and the preparation of the manuscript. BW participated in the statistical analyses and contributed to the preparation of the manuscript. EH-P developed the study concept and design, participated in the interpretation of the data and preparation of the manuscript. All authors read and approved the final manuscript.

\section{Competing interests}

The authors declare that they have no competing interests.

Received: 27 May 2011 Accepted: 24 June 2011 Published: 24 June 2011

\section{References}

1. Colledge NR, Wilson JA, Macintyre CCA, MacLennan WJ: The prevalence and characteristics of dizziness in an elderly community. Age Ageing 1994, 23:117-120.

2. Jönsson R, Sixt E, Landahl S, Rosenhall U: Prevalence of dizziness and vertigo in an urban elderly population. J Vestibular Res 2004, 14:47-52.

3. Grimby A, Rosenhall U: Health-related quality of life and dizziness in old age. Gerontology 1995, 41:286-298.

4. Tinetti ME, Williams CS, Gill TM: Dizziness among older adults: A possible geriatric syndrome. Ann Intern Med 2000, 132:337-344

5. Bird JC, Beynon GJ, Prevost AT, Baguely DM: An analysis of referral patterns for dizziness in the primary care setting. Br J Gen Pract 1998, 48:1828-1832.

6. Kroenke K, Lucas CA, Rosenberg ML, Scherokman B, Herbers JE, Wehrle PA, Boggi JO: Causes of persistent dizziness. Ann Intern Med 1992, 117:898-904.

7. Sloane PD, Hartman M, Mitchell CM: Psychological factors associated with chronic dizziness in patients aged 60 and older. J Am Geriatr Soc 1994, 42:847-852. 
8. Hanley K, O'Dowd T: Symptoms of vertigo in general practice: a prospective study of diagnosis. Br J Gen Pract 2002, 52:809-812.

9. Gassmann KG, Rupprecht R: Dizziness in an older community dwelling population: a multifactorial syndrome. J Nutr Health Aging 2009, 13:278-282.

10. Kao AC, Nanda A, Williams CS, Tinetti ME: Validation of Dizziness as a Possible Geriatric Syndrome. J Am Geriatr Soc 2001, 49:72-75.

11. Hsu LC, Hu HH, Wong WJ, Wang SJ, Luk YO, Chern CM: Quality of life in elderly patients with dizziness: analysis of the the Short-Form Health Survey in 197 patients. Acta Otolaryngol 2005, 125:55-59.

12. Wiltink J, Tschan R, Michal M, Subic-Wrana C, Eckhardt-Henn A, Dietrich M, Beutel ME: Dizziness: Anxiety, health care utilization and health behavior - results from a represantative German community survey. J Psychosom Res 2009, 66:417-424.

13. Tinetti ME, Williams CS, Gill TM: Health, functional, and psychological outcomes among older persons with chronic dizziness. J Am Geriatr Soc 2000, 48:417-421.

14. Newman-Toker DE, Dy FJ, Stanton VA, Zee DS, Calkins H, Robinson KA: How often is dizziness from primary cardiovascular disease true vertigo? A systematic review. J Gen Intern Med 2008, 23:2087-2094.

15. Sloane PD, Coeytaux RR, Beck RS, Dallara J: Dizziness: state of the science. Ann Intern Med 2001, 134:823-832.

16. Hoffmann RM, Einstadter D, Kroenke K: Evaluating dizziness. Am J Med 1999, 107:468-472.

17. Drachman DA, Hart CW: An approach to the dizzy patient. Neurology 1972, 22:323-334.

18. Jacobsen GP, Newman CW: The development of the Dizziness Handicap Inventory. Arch Otolaryngol Head Neck Surg 1990, 116:424-427.

19. Kruschinski C, Klaassen A, Breull A, Broll A, Hummers-Pradier : Priorities of elderly patients in general practice: Findings and psychometric properties of the "Dizziness Needs Assessment" (DiNA). Z Gerontol Geriatr 2010, 43:317-323.

20. Spitzer RL, Kroenke K, Linzer M, deGruy FV, Hahn SR, Brody D, Johnson JG: Utility of a new procedure for diagnosing mental disorders in primary care. The PRIME-MD 1000 study. JAMA 1994, 272:1749-56.

21. Shiekh J, Yesavage J: Geriatric Depression Scale; recent findings and development of a short version.Edited by: Brink T. Clinical Gerontology: a guide to assessment and intervention. Howarth Press, New York; 1986:.

22. Ware J, Kosinski M, Keller SD: A 12-Item Short-Form Health Survey: construction of scales and preliminary tests of reliability and validity. Med Care 1996, 34:220-233.

23. Fillenbaum GG: Screening the elderly. A brief instrumental activities of daily living measure. J Am Geriatr Soc 1985, 33:698-706.

24. Sloane PD, Dallara J, Roach C, Bailey KE, Mitchell M, McNutt R: Management of dizziness in primary care. J Am Board Fam Pract 1994, 7:1-8.

25. Mendel B, Bergenius J, Langius A: Dizziness symptom severity and impact on daily living as perceived by patients suffering from peripheral vestibular disorder. Clin Otolaryngol Allied Sci 1999, 24:286-293.

26. Kruschinski C, Theile G, Dreier SD, Hummers-Pradier E: Priorities of elderly patients suffering from dizziness: a qualitative study. Eur $J$ Gen Pract 2010, 16:6-11.

27. Katsarkas A: Dizziness in aging: the clinical experience. Geriatrics 2008, 63:18-20.

28. Maarsingh OR, Dros J, Schellevis FG, van Weert HC, Bindels PJ, van der Holst HE: Dizziness reported by elderly patients in family practice: prevalence, incidence, and clinical characteristics. BMC Fam Pract 2010, 11:2.

29. Hansson EE, Mansson NO, Hakansson A: Benign paroxysymal positional vertigo among elderly patients in primary health care. Gerontology 2005, 51:386-389.

30. Hilton M, Pinder D: The Epley (canalith repositioning) manoeuvre for benign paroxysymal positional vertigo. Cochrane Database Syst Rev 2004 2:CD003162.

31. Tamber AL, Wilhelmsen KT, Strand Ll: Measurement properties of the Dizziness Handicap Inventory by cross-sectional and longitudinal designs. Health Qual Life Outcomes 2009, 7:101.

\section{Pre-publication history}

The pre-publication history for this paper can be accessed here:

http://www.biomedcentral.com/1471-2296/12/58/prepub doi:10.1186/1471-2296-12-58

Cite this article as: Sczepanek et al:: Newly diagnosed incident dizziness of older patients: a follow-up study in primary care. BMC Family Practice 2011 12:58

\section{Submit your next manuscript to BioMed Central and take full advantage of:}

- Convenient online submission

- Thorough peer review

- No space constraints or color figure charges

- Immediate publication on acceptance

- Inclusion in PubMed, CAS, Scopus and Google Scholar

- Research which is freely available for redistribution 